\title{
Robust take-off and landing for a quadrotor vehicle
}

\author{
D. Cabecinhas, R. Naldi, L. Marconi, C. Silvestre, R. Cunha
}

\begin{abstract}
This paper addresses the problem of robust takeoff and landing control of a quadrotor UAV (Unmanned Aerial Vehicle). During the critical flight phases of takeoff and landing the vehicle dynamics change according to the possible existence of contact with the ground. To model the vehicle during the overall maneuver a hybrid automaton is used where each state corresponds to a different dynamic behavior exhibited by the UAV. The original takeoff and landing problems are then addressed as a problem of tracking suitable reference signals in order to achieve the desired transitions between different hybrid states of the automaton. Both reference trajectories and feedback control laws are derived to explicitly account for measurement noise and uncertainties, in both the environment and in the vehicle dynamics. Simulation results demonstrate the effectiveness of the proposed solution.
\end{abstract}

\section{INTRODUCTION}

Flight control of autonomous Unmanned Aerial Vehicles (UAV) is an active and extensively researched topic, with crucial importance to numerous civilian and military applications [1], [2], [3], [4], [5]. To be truly autonomous, an UAV must perform autonomously maneuvers that encompass not only the normal flight conditions, like hover or forward flight, but also the critical takeoff and landing maneuvers, where interaction with the ground occurs.

The critical takeoff and landing phases of flight are of fundamental importance since they are performed by all aerial vehicles. In most practical settings, the controller must provide robustness to uncertainties in both the environment and the dynamical vehicle model. The methodology adopted to addressed this problem borrows from the control framework proposed in [6]. In this approach, the vehicle is modeled as an hybrid automaton where each state corresponds to a different operating condition, described by different dynamics, according to the nature of the ground contact.

Hybrid automata allow for a complex model to be described in a modular way by collecting simpler dynamical models, each one focusing only on a precise operating mode of the system. They constitute a subset of the larger class of hybrid dynamical systems, which is nowadays an important and active topic of research [7], [8], [9], [10]. Hybrid dynamical systems were recently the subject of an in-depth article [11] focusing on modeling their dynamics, on elements of stability theory, and on the basics of hybrid control.

This work was partially supported by Fundação para a Ciência e a Tecnologia (ISR/IST plurianual funding) and by the project PTDC/EEAACR/72853/2006 HELICIM of the FCT and AIRTICI from AdI.

The work of D. Cabecinhas was supported by a PhD Student Grant from the FCT POCTI program, SFRH / BD / 31439 / 2006.

D. Cabecinhas, R. Cunha and C. Silvestre are with Institute for Systems and Robotics, Instituto Superior Técnico, Portugal.

R. Naldi and L. Marconi are with CASY-DEIS, University of Bologna, Italy.
Once the hybrid automaton is defined, the takeoff and landing control problems are addressed as tracking control problems. In particular both the reference signals, which are such that their practical and not perfect tracking guarantees the desired transitions to happen, and the feedback laws for each operating mode are derived considering explicitly the presence of uncertainties and measurement noise. The latter in particular affects the knowledge of the current operating mode, which can be determined using contact or force sensors placed at each extremity of the vehicles landing gear. The control law is therefore required to be robust to mismatches between actual and detected operating mode resulting from sensor noise.

The main contribution of this work consists in the explicit design of the hybrid automaton and takeoff and landing controller for a quadrotor vehicle, which guarantees robustness to model and environment uncertainties and to sensor noise in the operating mode detection.

\section{QUADROTOR HYBRID MODEL}

The UAV considered in this paper is a quadrotor aircraft actuated in force generated by propellers. For sake of simplicity, we consider only the "planar dynamics" on the configuration manifold $\mathbb{S}^{1} \times \mathbb{R}^{2}$ since the general "spatial dynamics", defined on the configuration manifold $S O(3) \times$ $\mathbb{R}^{3}$, can be dealt with in a similar way, though heavier from a notational viewpoint.

During take-off and landing maneuvers the vehicle dynamics can vary greatly, depending on the type of contact with the ground. To accurately describe the behaviour of the rotorcraft during a complete takeoff and landing maneuver we use the hybrid automata framework. The different states of the hybrid automata correspond to each of the three operating modes considered for the vehicle, related to the number of points of contact of the UAV with the ground. For the sake of simplicity we consider the ground to be a horizontal surface at a height $\beta$. The physical state of the quadrotor is described by the center of mass coordinates $(x, z)$, angle with the horizontal $\theta$, and respective derivatives. For the operation modes where contact with the ground exists it is simpler to consider the horizontal displacement $\alpha$ of the contact point rather than the horizontal displacement $x$ of the center of mass. They are related by

$$
\alpha=x+\ell \cos (\theta+\gamma) \text {. }
$$

Due to the quadrotor symmetry we consider only takeoff and landing maneuvers where rotation occurs around the contact point $\mathrm{P}_{2}$, resulting in $\theta \geq 0$. The symmetric situation is dealt similarly and will not be discussed in this paper. 


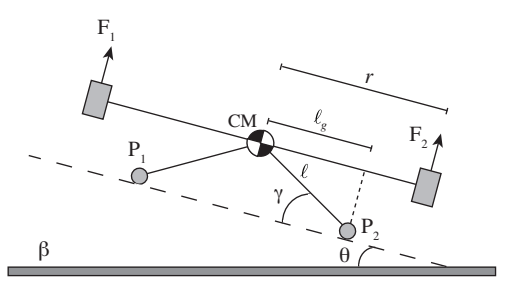

Fig. 1. Planar quadrotor description

\section{A. Dynamics of operating modes}

1) Free Flight: In this operating mode the aircraft is completely airborne and there is no contact with the ground. To model the free flight mode we use the standard equations of motion considering atmospheric drag, where $m$ and $J$ denote respectively the mass and moment of inertia of the vehicle, $g$ the gravity acceleration and $\lambda_{x}, \lambda_{z}$ the first order aerodynamic drag coefficients.

$$
\begin{aligned}
m \ddot{x} & =\left(F_{1}+F_{2}\right) \sin \theta-\lambda_{x} \dot{x} \\
m \ddot{z} & =\left(F_{1}+F_{2}\right) \cos \theta-m g-\lambda_{z} \dot{z} \\
J \ddot{\theta} & =\left(F_{1}-F_{2}\right) r
\end{aligned}
$$

The value of the drag coefficients $\lambda_{x}, \lambda_{z}$ are subject to possibly large uncertainties.

2) Partial interaction with the ground: In this mode of operation there is only one contact point of the quadrotor with the ground. The equations of motion for the partial interaction mode are derived from the Lagrangian for the system in that configuration given by

$$
\mathcal{L}=\frac{1}{2} m\left(\dot{x}^{2}+\dot{z}^{2}\right)-m g \ell \sin (\theta+\gamma)
$$

The obtained equations of movement, noting (1), can be written as

$$
\begin{aligned}
& m \ddot{\alpha}+m \ell \ddot{\theta} \sin (\theta+\gamma)+ m \ell \dot{\theta}^{2} \cos (\theta+\gamma)= \\
&\left(F_{1}+F_{2}\right) \sin \theta-\lambda_{H} \dot{\alpha}, \\
& m \ell \ddot{\alpha} \sin (\theta+\gamma)+m \ell^{2} \ddot{\theta}+m g \ell \cos (\theta+\gamma)= \\
&\left(F_{1}+F_{2}\right) \ell_{g}+\left(F_{1}-F_{2}\right) r
\end{aligned}
$$

where $\lambda_{H}$ is the horizontal surface friction coefficient. For sake of simplicity friction is modeled through a stateless linear model affected by large uncertainties on the coefficients.

For angles $\theta<\pi / 2-\gamma$, (3) can be written as the decoupled dynamic equations

$$
\begin{gathered}
\ddot{\alpha}=F_{\alpha}+h_{\alpha}\left(\theta, \dot{\theta}, \dot{\alpha}, \lambda_{H}\right), \\
\ddot{\theta}=F_{\theta}+h_{\theta}\left(\theta, \dot{\theta}, \dot{\alpha}, \lambda_{H}\right),
\end{gathered}
$$

where the functions $h_{\alpha}$ and $h_{\theta}$ are given by

$h_{\alpha}\left(\theta, \dot{\theta}, \dot{\alpha}, \lambda_{H}\right)=g \tan (\theta+\gamma)-\frac{\ell}{\cos (\theta+\gamma)} \dot{\theta}^{2}-\frac{\lambda_{H}}{m \cos ^{2}(\theta+\gamma)} \dot{\alpha}$,

$h_{\theta}\left(\theta, \dot{\theta}, \dot{\alpha}, \lambda_{H}\right)=-\frac{g}{\ell \cos (\theta+\gamma)}+\tan (\theta+\gamma) \dot{\theta}^{2}+\frac{\lambda_{H} \sin (\theta+\gamma)}{m \ell \cos ^{2}(\theta+\gamma)} \dot{\alpha}$.

and there is a one-to-one correspondence between the virtual inputs $F_{\theta}, F_{\alpha}$ and the real inputs $F_{1}$ and $F_{2}$. The equivalent equations (3) and (4) describe a 4-state dynamical model for the vehicle. The vertical coordinate of the center of mass and its derivative are uniquely defined by $\theta$ and $\dot{\theta}$ through

$$
\begin{aligned}
& z=\beta+\ell \sin (\theta+\gamma) \\
& \dot{z}=\dot{\theta} \ell \cos (\theta+\gamma),
\end{aligned}
$$

where the constant $\beta$ is the height of the ground.

3) Complete interaction with the ground: In this state the vehicle is completely landed and only the ground contact drag affects the motion of the vehicle. The dynamic model is completely described by the 2-state dynamical system

$$
m \ddot{\alpha}=-\lambda_{H} \dot{\alpha} .
$$

\section{B. Hybrid dynamical model of the overall dynamics}

A description of the overall dynamics is obtained by means of a hybrid automaton whose hybrid states correspond to the above described operating modes. In this section we define rigorously the automaton for the specific quadrotor application. A hybrid automaton is identified by the following objects, instanced here for the specific case of the planar quadrotor.

1) Operating Modes: A set $\mathcal{Q}$ of operating modes, denoted by $\mathcal{Q}=\{L L, T L, F F\}$, with the meaning

- LL, Landed, when the aircraft is completely landed and has two ground contact points;

- TL, Take-off and Landing, in this situation there exist only one contact point of the UAV with the ground;

- FF, Free Flight, situation where the aircraft is completely airborne and there is no contact with the ground.

2) Domain mapping: The state of the system $\xi \in$ $\mathbb{R}^{6}$ is described by either of the vectors $(x, \dot{x}, z, \dot{z}, \theta, \dot{\theta})$, $(\alpha, \dot{\alpha}, z, \dot{z}, \theta, \dot{\theta})$. When the UAV is in contact with the ground (LL or TL operating modes) the preferred notation is using $\alpha, \dot{\alpha}$ while $x, \dot{x}$ is preferred for the free flight mode. In the work it is assumed that the planar UAV is controlled through two inputs $F_{1}, F_{2}$, which correspond to the forces generated by the propellers. These are bounded by a minimum and maximum value, leading to the definition of the input domain $U \in \mathbb{R}^{2}$ as the compact interval $U=\left[F_{\min }, F_{\max }\right] \times$ $\left[F_{\min }, F_{\max }\right]$. The domain mapping $\mathcal{D}: \mathcal{Q} \rightrightarrows \mathbb{R}^{6} \times \mathbb{R}^{2}$ defines, for each operating mode, the set of values where the state $\xi$ and the control input $u$ may range.

$$
\begin{aligned}
\mathcal{D}(F F)= & \mathbb{R}^{6} \times U, \\
\mathcal{D}(T L)= & \left\{\xi \in \mathbb{R}^{6}: z=\beta+\ell \sin (\theta+\gamma),\right. \\
& \theta<\pi / 2-\gamma\} \times U, \\
\mathcal{D}(L L)= & \left\{\xi \in \mathbb{R}^{6}: z=\beta+\ell \sin (\gamma), \theta=0\right\} \times U .
\end{aligned}
$$

3) Flow map: The flow map $f: \mathcal{Q} \times \mathbb{R}^{6} \times \mathbb{R}^{2} \rightarrow \mathbb{R}^{6}$ describes for each operating mode $q \in\{L L, T L, F F\}$ the evolution of the state variables. In each operating mode $q$ we have the dynamic system $\dot{\xi}=f(q, \xi, u)$, where each function $f(q, \xi, u)$ is derived from the differential equations (2), (3) and (7) presented in the previous section.

4) Edges: The set of edges $\mathcal{E} \subset \mathcal{Q} \times \mathcal{Q}$ includes all the pairs $\left(q_{1}, q_{2}\right)$ such that a transition between the modes $q_{1}$ and $q_{2}$ is possible, for some combination of state and actuation. For the takeoff and landing procedure we consider 
that transitions are possible between every state. The set of edges is thus composed of

$$
\begin{aligned}
& \{L L, T L\}, \quad\{T L, L L\}, \quad\{F F, L L\}, \\
& \{L L, F F\}, \quad\{T L, F F\}, \quad\{F F, T L\} .
\end{aligned}
$$

5) Guard mapping: The guard mapping $\mathcal{G}: \mathcal{E} \rightrightarrows \mathbb{R}^{6} \times \mathbb{R}^{2}$ determines, for each $\left(q_{1}, q_{2}\right) \in \mathcal{E}$, the set $\mathcal{G}\left(\left\{q_{1}, q_{2}\right\}\right)$ that the quadrotor state $\xi$ and inputs $F_{1}, F_{2}$ must belong so that a transition from the hybrid mode $q_{1}$ to $q_{2}$ can occur. To simplify notation we introduce two functions,

$F_{Z}\left(\theta, F_{1}, F_{2}\right)=\left(F_{1}+F_{2}\right) \cos \theta-m g$, $F_{\tau}\left(\theta, F_{1}, F_{2}\right)=\left(F_{1}+F_{2}\right) l_{g}+\left(F_{1}-F_{2}\right) r-m g \ell \cos (\theta+\gamma)$ which denote the resulting force acting on the vehicle along the $z$-axis and the resulting torque around the contact point $P_{2}$, respectively. Transitions to and from the FF operating mode are based on the sign of $F_{Z}$ and switches between the LL and TL modes are governed by the sign of $F_{\tau}$. The guard sets for the quadrotor hybrid automaton are the following.

$$
\begin{aligned}
& \mathcal{G}(\{L L, T L\})=\left\{(\xi, u) \in \mathcal{D}(L L): F_{\tau}\left(\theta, F_{1}, F_{2}\right) \geq 0 \vee\right. \\
&\theta>0\} \\
& \mathcal{G}(\{L L, F F\})=\left\{(\xi, u) \in \mathcal{D}(L L): F_{Z}\left(\theta, F_{1}, F_{2}\right) \geq 0\right\} \\
& \mathcal{G}(\{T L, L L\})=\{(\xi, u) \in \mathcal{D}(T L): \theta \leq 0 \wedge \\
&\left.F_{\tau}\left(\theta, F_{1}, F_{2}\right)<0\right\} \\
& \mathcal{G}(\{T L, F F\})=\left\{(\xi, u) \in \mathcal{D}(T L): F_{Z}\left(\theta, F_{1}, F_{2}\right) \geq 0\right\} \\
& \mathcal{G}(\{F F, L L\})=\{(\xi, u) \in \mathcal{D}(F F): \theta=0 \wedge \\
&\left.z \leq \beta+\ell \sin (\gamma) \wedge F_{Z}(\theta, F 1, F 2)<0\right\} \\
& \mathcal{G}(\{F F, T L\})=\{(\xi, u) \in \mathcal{D}(F F): z \leq \beta+\ell \sin (\theta+\gamma) \wedge \\
&\left.F_{Z}(\theta, F 1, F 2)<0\right\}
\end{aligned}
$$

6) Reset map: For each $\left(q_{1}, q_{2}\right) \in \mathcal{E}$ and $(\xi, u) \in$ $\mathcal{G}\left(\left\{q_{1}, q_{2}\right\}\right)$, the reset map $\mathcal{R}: \mathcal{E} \times \mathbb{R}^{6} \times \mathbb{R}^{2} \rightarrow \mathbb{R}^{6}$ identifies the jump of the state variable $\xi$ during the operating mode transition from $q_{1}$ to $q_{2}$. The jumps in the state reflect instant changes due to collisions with the ground and also to the use of either $\alpha$ or $x$ to describe the state, according to the operating mode. The collisions with the ground are modeled as being perfectly inelastic, resulting in the reset maps

$$
\begin{aligned}
\mathcal{R}(\{L L, T L\},(\xi, u)) & =\xi, \\
\mathcal{R}(\{F F, T L\},(\xi, u)) & =\left(\alpha, \dot{\alpha}, z, \dot{z}, \theta, \frac{\dot{z}}{\ell \cos (\theta+\gamma)}\right), \\
\mathcal{R}(\{*, F F\},(\xi, u)) & =(x, \dot{x}, z, \dot{z}, \theta, \dot{\theta}), \\
\mathcal{R}(\{*, L L\},(\xi, u)) & =(\alpha, \dot{\alpha}, z, 0,0,0) .
\end{aligned}
$$

\section{The TAKeoff AND LANDing Control FRAMEWORK}

The takeoff and landing problem is divided into two steps. Foremost, reference maneuvers (encompassing the vehicle state trajectory and control inputs) are designed with the objective of maintaining a certain operating mode or switching from one operating mode to the other robustly, i.e. a non perfect tracking of these maneuvers does not lead to undesired operating mode changes. Once the maneuvers are conceived, control laws are designed to guarantee "sufficiently close" following of the desired maneuver.
Both the definitions of the references and the design of the closed-loop control laws will rely upon a parameter, $\epsilon$, which in practice specifies how far the actual trajectory can be with respect to the desired one.

To precisely define the maneuvers of interest, we denote by $v_{q}(t)=(\xi(t), u(t))$ a maneuver taking place in the operating mode $q \in \mathcal{Q}$ and by $\operatorname{gr} v_{q}$ the graph of the maneuver in a certain time interval. According to [6], towards which the reader is referred for further mathematical details, three different types of maneuvers are defined. The first one, which is denoted as $\epsilon$-robust $q_{1}$-single maneuver in $\left[t_{0}, t_{1}\right)$, is such that the state and the input do not intersect any guard condition in order to maintain the same "single" operating mode $q_{1}$. The second type, denoted as $\epsilon$-robust $q_{1} \rightarrow q_{2}$ approach maneuver in $\left[t_{0}, T\right]$, is such that at time $T$ the maneuver belongs to the desired guard set, $\mathcal{G}\left(\left\{q_{1}, q_{2}\right\}\right)$, in order to switch to the operating mode $q_{2}$. The last one, the $q_{1} \rightarrow q_{2}$ transition maneuver in $\left[t_{0}, t_{1}\right)$, is obtained as a combination of an $\epsilon$-robust $q_{1} \rightarrow q_{2}$ approach maneuver and a set of $\epsilon$-robust $q_{2}$-single maneuvers.

\section{Robust Maneuvers}

In this section we actually build some reference maneuvers for the particular case of the quadrotor, with a focus on maneuvers involving both the TL and FF operating modes. In what follows, the subscript "sp" on a set denotes that it is obtained considering the span of all the possible values for the uncertainties affecting it. Although uncertainties can be considered in every parameter of the vehicle, to keep the analysis to a tractable level we consider only uncertainties in the drag coefficients $\lambda_{x}$ and $\lambda_{z}$ on the FF mode and the ground drag coefficient $\lambda_{H}$ when in the TL operating mode. Their nominal values are denoted by $\lambda_{x 0}, \lambda_{z 0}$ and $\lambda_{H 0}$, respectively. Moreover we assume that the ground height $\beta$ is not perfectly known and that the controller is only allowed to know an upper bound denoted by $\bar{\beta}$.

\section{A. $\epsilon$-robust TL-single reference maneuver}

Nominal reference maneuvers are obtained through system inversion, with the reference inputs being computed from the desired state trajectories. Given twice differentiable desired state trajectories $\theta^{\star}(t), \alpha^{\star}(t)$, the nominal inversion of the system (4) results in

$$
\begin{aligned}
& F_{\alpha}^{\star}(t)=\ddot{\alpha}^{\star}(t)-h_{\alpha}\left(\theta^{\star}(t), \dot{\theta}^{\star}(t), \dot{\alpha}^{\star}(t), \lambda_{H 0}\right) \\
& F_{\theta}^{\star}(t)=\ddot{\theta}^{\star}(t)-h_{\theta}\left(\theta^{\star}(t), \dot{\theta}^{\star}(t), \dot{\alpha}^{\star}(t), \lambda_{H 0}\right) .
\end{aligned}
$$

Additionally, if $\theta^{\star}(t)<\pi / 2-\gamma$ and $\theta^{\star}(t) \neq 0$, the reference actuation values can be recovered from the "virtual" forces through

$$
\left(\begin{array}{l}
F_{1}^{\star}(t) \\
F_{2}^{\star}(t)
\end{array}\right)=G^{-1}\left(\theta^{\star}(t)\right) L\left(\theta^{\star}(t)\right)\left(\begin{array}{l}
F_{\alpha}^{\star}(t) \\
F_{\theta}^{\star}(t)
\end{array}\right)
$$

where matrices $G(\theta)$ and $L(\theta)$ are expressed as

$$
G(\theta)=\left(\begin{array}{cc}
\sin \theta & \sin \theta \\
\ell_{g}+r & \ell_{g}-r
\end{array}\right), \quad L(\theta)=m\left(\begin{array}{cc}
1 & \ell \sin (\theta+\gamma) \\
\ell \sin (\theta+\gamma) & \ell^{2}
\end{array}\right) .
$$

In an $\epsilon$-robust TL maneuver, the desired trajectory $\theta^{\star}(t)$ and the resulting forces $F_{1}^{\star}(t), F_{2}^{\star}(t)$ are such that

$$
\begin{array}{r}
F_{Z}\left(\theta(t), F_{1}(t), F_{2}(t)\right)<0 \\
\theta(t)>0 \vee F_{\tau}\left(\theta(t), F_{1}(t), F_{2}(t)\right)>0
\end{array}
$$


for all $\left(\theta(t), F_{1}(t), F_{2}(t)\right) \epsilon$-close to $\left(\theta^{\star}(t), F_{1}^{\star}(t), F_{2}^{\star}(t)\right)$ and for all the possible uncertainties in the guard sets.

Note that as the angle $\theta$ approaches zero, $\sin \theta$ also approaches zero and the matrix $G(\theta)$ becomes almost singular, leading to conditioning problems when recovering $F_{1}, F_{2}$ using (10). For angles $\theta$ close to zero this controller is avoided and the control effort is shifted towards controlling the angle $\theta$, ignoring the horizontal displacement $\alpha$. This results in definition of $\left(F_{1}-F_{2}\right)^{\star}$ through (11) and of $\left(F_{1}+F_{2}\right)^{\star}$ being regarded as a design parameter.

$$
F_{\theta}^{\star}=\frac{\left(F_{1}+F_{2}\right)^{\star}\left(\ell_{g}-\ell \sin \left(\theta^{\star}\right) \sin \left(\theta^{\star}+\gamma\right)\right)+\left(F_{1}-F_{2}\right)^{\star} r}{m \ell^{2} \cos ^{2}\left(\theta^{\star}+\gamma\right)} .
$$

\section{$B$. $\epsilon$-robust $F F$-single reference maneuver}

Starting with class $C^{4}$ state trajectories, $x^{\star}(t), z^{\star}(t)$ and inverting system (2) the resulting reference angle $\theta^{\star}(t)$ and control forces $\left(F_{1}^{\star}(t), F_{2}^{\star}(t)\right)$ are given by

$$
\begin{aligned}
\theta^{\star}(t) & =\tan ^{-1}\left(\frac{m \ddot{x}^{\star}(t)+\lambda_{x 0} \dot{x}^{\star}(t)}{m g+m \ddot{z}^{\star}(t)+\lambda_{x 0} \dot{z}^{\star}(t)}\right), \\
F_{1}^{\star}(t)+F_{2}^{\star}(t) & =\frac{m g+m \ddot{z}^{\star}(t)+\lambda_{x 0} \dot{z}^{\star}(t)}{\cos \theta^{\star}(t)}, \\
F_{1}^{\star}(t)-F_{2}^{\star}(t) & =\frac{J}{r} \ddot{\theta}^{\star}(t) .
\end{aligned}
$$

As a robust maneuver in the FF state, the reference trajectory verifies the inequality

$$
z(t)>\bar{\beta}+\ell \sin (\theta(t)+\gamma) \vee F_{Z}\left(\theta(t), F_{1}(t), F_{2}(t)\right)>0
$$

for all $\quad\left(z(t), \theta(t), F_{1}(t), F_{2}(t)\right) \quad \epsilon$-close $\quad$ to $\left(z^{\star}(t), \theta^{\star}(t), F_{1}^{\star}(t), F_{2}^{\star}(t)\right)$ and for all possible uncertainties in the guard set.

Introducing actuation limits, we have that $x^{\star}(t)$ and $z^{\star}(t)$ are chosen such that

$$
\frac{1}{2}\left(\frac{m g+m \ddot{z}^{\star}(t)+\lambda_{x 0} \dot{z}^{\star}(t)}{\cos \theta^{\star}(t)} \pm \frac{J}{r} \ddot{\theta}^{\star}(t)\right) \in\left[F_{\text {min }}, F_{\text {max }}\right]
$$

considering all possible uncertainties.

\section{C. $\epsilon$-robust $T L \rightarrow F F$ reference approach maneuver}

A $\mathrm{TL} \rightarrow \mathrm{FF}$ approach maneuver in $\left[t_{0}, T\right]$ is $\epsilon$-robust if the reference trajectory is robustly far from any guard condition except the desired one and the following is observed ${ }^{1}$

$$
B_{\epsilon}\left(v_{T L}^{\star}(T)\right) \subset\left\{F_{Z}\left(\theta, F_{1}, F_{2}\right) \geq 0\right\},
$$

which amounts to entering the desired guard condition robustly in a time upper-bounded by $T$.

Model inversion is used as for the $\epsilon$-robust TL maneuvers to determine the reference controls $F_{1}^{\star}(t)$ and $F_{2}^{\star}(t)$. Reference trajectories with sufficiently high reference horizontal acceleration $\ddot{\alpha}^{\star}(t)$ lead to (12) being verified.

\section{D. $\epsilon$-robust $F F \rightarrow T L$ reference approach maneuver}

For the sake of simplicity, as approach maneuvers we consider trajectories of constant vertical velocity $\dot{z}^{\star}(t)=$ $-\bar{v}_{z}$ and constant lateral position $x^{\star}(t)=\bar{x}$. From the model inversion, we get as reference trajectory and inputs

$$
\theta^{\star}(t)=0, \quad F_{1}^{\star}(t)=F_{2}^{\star}(t)=\frac{1}{2}\left(m g-\lambda_{z 0} \bar{v}_{z}\right) .
$$

The total vertical reference force is given by

$$
F_{Z}^{\star}(t)=F_{1}^{\star}(t)+F_{2}^{\star}(t)-m g=-\lambda_{z 0} \bar{v}_{z}
$$

\footnotetext{
${ }^{1}$ For a point $x \in R^{n}, B_{\epsilon}(x)$ denotes the ball of radius $\epsilon$ centered at $x$.
}

so that, as soon as the vehicle touches the ground, the maneuver belong to the desired guard set $\mathcal{G}(\{\mathrm{FF}, \mathrm{TL}\})$, i.e. $F_{Z}(\cdot)<0$, enabling the transition. This condition can be enforced more robustly by choosing larger values of $\bar{v}_{z}$.

\section{E. $\epsilon$-robust $T L \rightarrow L L$ reference approach maneuver}

From model inversion in the TL operating mode it turns out that the condition to be robustly in the desired operating mode, $B_{\epsilon}\left(v^{\star}(T)\right) \subset\left\{\theta \leq 0 \wedge F_{\tau}\left(\theta, F_{1}, F_{2}\right) \leq 0\right\}$, is satisfied by maneuvers such that the reference state trajectory $\theta^{\star}(t)$ goes to negative values with a sufficiently negative second derivative, leading to a negative angular acceleration.

\section{$F$. $T L \rightarrow F F$ and $F F \rightarrow T L$ reference transition maneuvers}

In the proposed framework, takeoff (landing) is obtained by tracking a $\mathrm{TL} \rightarrow \mathrm{FF}(\mathrm{FF} \rightarrow \mathrm{TL}$ ) reference transition maneuver. By construction a reference transition maneuver is built by considering an approach maneuver followed by a set of single maneuvers. The role of the set of single reference maneuvers is to have at least one of them close to the initial conditions in the new operating mode so that the tracking error can remain bounded and lower than the design parameter $\epsilon$. In this respect, the reference single maneuvers are designed once the set of all possible initial conditions after the switch of operating mode is estimated. In the case of the takeoff maneuver this set can be estimated considering the intersection of the approach maneuver with the guard set $\mathcal{G}(\{T L, F F\})$ (or $\mathcal{G}(\{F F, T L\})$ ). For the case of the $\mathrm{FF} \rightarrow \mathrm{TL}$ maneuver, the effect of the reset map $\mathcal{R}(\{F F, T L\})$ also has to be taken into account.

\section{Controller Design}

We now describe the local controllers designed for each operating mode to track the reference maneuvers defined before guaranteeing a tracking error lower or equal than $\epsilon$ despite the presence of parametric uncertainties. We consider two controllers for the TL mode, one that only tracks the reference trajectory angle and another that tracks both the reference angle and the horizontal displacement. When in the free flight mode the controller used is the one proposed in [12]. Hysteresis is considered when switching between the two controllers for the TL operating mode. This guarantees that chattering between these two controllers does not occur when the maneuver is tracked robustly.

\section{A. Control of $\theta$ and $\alpha$ in TL operating mode}

The dynamic equations governing the evolution of the horizontal displacement $\alpha$ and angle $\theta$ are given in (4). Considering the control inputs in those equations as being the nominal reference controls derived in (8) and (9) with added error terms

$$
F_{\alpha}=F_{\alpha}^{\star}+\tilde{F}_{\alpha}, \quad F_{\theta}=F_{\theta}^{\star}+\tilde{F}_{\theta},
$$

leads to the following vehicle error dynamics

$$
\begin{aligned}
& \ddot{\tilde{\alpha}}=\tilde{F}_{\alpha}+\Psi_{\alpha}(\tilde{\theta}, \dot{\tilde{\theta}}, \dot{\tilde{\alpha}}, t)+\delta_{\alpha}(t), \\
& \ddot{\tilde{\theta}}=\tilde{F}_{\theta}+\Psi_{\theta}(\tilde{\theta}, \dot{\tilde{\theta}}, \dot{\tilde{\alpha}}, t)+\delta_{\theta}(t),
\end{aligned}
$$


where

$$
\begin{aligned}
& \Psi_{\alpha}(\tilde{\theta}, \dot{\tilde{\theta}}, \dot{\tilde{\alpha}}, t)= h_{\alpha}\left(\theta^{\star}+\tilde{\theta}, \dot{\theta}^{\star}+\dot{\tilde{\theta}}, \dot{\alpha}^{\star}+\dot{\tilde{\alpha}}, \lambda_{H}\right) \\
&-h_{\alpha}\left(\theta^{\star}, \dot{\theta}^{\star}, \dot{\alpha}^{\star}, \lambda_{H}\right), \\
& \Psi_{\theta}(\tilde{\theta}, \dot{\tilde{\theta}}, \dot{\tilde{\alpha}}, t)= h_{\theta}\left(\theta^{\star}+\tilde{\theta}, \dot{\theta}^{\star}+\dot{\tilde{\theta}}, \dot{\alpha}^{\star}+\dot{\tilde{\alpha}}, \lambda_{H}\right) \\
&-h_{\theta}\left(\theta^{\star}, \dot{\theta}^{\star}, \dot{\alpha}^{\star}, \lambda_{H}\right), \\
& \delta_{\alpha}(t)= \frac{\lambda_{H}-\lambda_{H 0}}{m} \dot{\alpha}^{\star} \frac{\lambda_{H}}{m \cos ^{2}\left(\theta^{\star}+\gamma\right)}, \\
& \delta_{\theta}(t)=-\frac{\lambda_{H}-\lambda_{H 0}}{m} \dot{\alpha}^{\star} \frac{\lambda_{H} \sin \left(\theta^{\star}+\gamma\right)}{m l \cos ^{2}\left(\theta^{\star}+\gamma\right)},
\end{aligned}
$$

and functions $h_{\alpha}(\cdot)$ and $h_{\theta}(\cdot)$ are defined in (5) and (6). We control the error dynamics (14) using PD control laws for the error term of both virtual forces $\tilde{F}_{\alpha}$ and $\tilde{F}_{\theta}$

$$
\tilde{F}_{\alpha}=-K_{P_{\alpha}}\left(\tilde{\alpha}+K_{D_{\alpha}} \dot{\tilde{\alpha}}\right), \quad \tilde{F}_{\theta}=-K_{P_{\theta}}\left(\tilde{\theta}+K_{D_{\theta}} \dot{\tilde{\theta}}\right) .
$$

With proper tuning of the parameters $K_{P_{i}}$ and $K_{D_{i}}, i=$ $\{\alpha, \theta\}$, we design a closed-loop error system which is Inputto-State Stable (ISS) with restrictions on the initial state and disturbances [13] while guaranteeing well-definiteness of the original controls $F_{1}, F_{2}$. We state the result in the following Proposition (proofs are omitted here for reason of space).

Proposition 1: Let $c_{1}>0$ such that $\left\|\theta^{\star}\right\|_{\infty}+c_{1} \leq \pi / 2-\gamma$ and let $c_{2}, \Delta$ and $K_{D_{i}}$ be arbitrary positive numbers, for $i=\{\alpha, \theta\}$. There exists $K_{P_{i}}^{\star}>0$ such that for all $K_{P_{i}} \geq$ $K_{P_{i}}^{\star}$ the closed-loop of system (14) with controller (15) is ISS with restrictions $c_{1}$ on the initial state $\tilde{\theta}(0)$, restriction $c_{2}$ on the initial states $\dot{\tilde{\theta}}(0)$ and $(\tilde{\alpha}, \dot{\tilde{\alpha}})$ and restriction $\Delta$ on the disturbances $\delta_{\theta}$ and $\delta_{\theta}$. Furthermore, the restrictions restrictions are such that the closed-loop trajectory verifies $\xi(t)-\xi^{\star}(t)<\epsilon$ and $\theta(t)<\pi / 2-\gamma-\epsilon$ for all $t \geq 0$.

\section{B. Control of $\theta$ in the TL mode}

For small $\theta$ angles the control objective is to track a desired trajectory $\theta^{\star}(t)$ while being robust to external disturbances. The dynamic equation governing the angle $\theta$ in the TL operating mode is (9), with the reference input given by (11) and $\dot{\alpha}^{\star}$ dependent on the design parameter function $\left(F_{1}(t)+F_{2}(t)\right)^{\star}$. The control approach for $\theta$ is identical to the one previously presented for control of both $\alpha$ and $\theta$. We use (13), (15) and recover the actuation $\left(F_{1}(t)-F_{2}(t)\right)$ from $F_{\theta}(t)$ and $\left(F_{1}(t)+F_{2}(t)\right)$ by means of the equivalent of (11) for real (and not reference) trajectories. As before, ISS with restrictions on the initial state and disturbances is achieved, for the angle $\theta$.

\section{Control in Free Flight}

To control the quadrotor vehicle in free-flight we adopt an inner-loop control strategy based on the controller proposed in [12]. The controller renders the closed-loop system ISS with restrictions on initial conditions. We define new control inputs $u, v$ that are in a 1-to-1 relationship with the quadrotor's real inputs,

$$
u=\left(F_{1}+F_{2}\right) \cos \theta, v=F_{1}-F_{2} .
$$

The error dynamic system that results from acting on the vehicle with controls $v=v^{\star}+\tilde{v}, u=u^{\star}+\tilde{u}$ where $u^{\star}=$

$$
\begin{aligned}
& \left(F_{1}^{\star}+F_{2}^{\star}\right) \cos \theta^{\star}, v^{\star}=F_{1}^{\star}-F_{2}^{\star} \text { is } \\
& m \ddot{\tilde{x}}=\left(\tan \left(\tilde{\theta}+\theta^{\star}\right)-\tan \theta^{\star}\right) u^{\star}-\lambda_{x} \dot{\tilde{x}}+\tan \theta \tilde{u}+\delta_{x}(t), \\
& m \ddot{\tilde{z}}=\tilde{u}-\lambda_{z} \dot{\tilde{z}}+\delta_{z}(t), \quad J \tilde{\tilde{\theta}}=r \tilde{v},
\end{aligned}
$$

with the disturbances given by $\delta_{x}(t)=\left(\lambda_{x 0}-\lambda_{x}\right) \dot{x}^{\star}(t)$, $\delta_{z}(t)=\left(\lambda_{z 0}-\lambda_{z}\right) \dot{z}^{\star}(t)$. The ISS result for (16) is summarized in the following Proposition.

Proposition 2: Consider the following control law for the error system (16)

$$
\begin{aligned}
\tilde{u} & =-k_{1} \tilde{z}-k_{2} \dot{\tilde{z}} \\
\tilde{v} & =-K_{P}\left(K_{D} \dot{\tilde{\theta}}+\tan \left(\tilde{\theta}+\theta^{\star}(t)\right)-\tan \theta^{\star}(t)+\theta_{\text {out }}\right) \\
\theta_{\text {out }} & =\lambda_{2} \sigma\left(\frac{K_{2}}{\lambda_{2}} \xi_{2}\right) \quad \xi_{2}=\dot{\tilde{x}}+\lambda_{1} \sigma\left(\frac{K_{1}}{\lambda_{1}} \tilde{x}\right)
\end{aligned}
$$

where $\sigma$ is a saturation function. There are positive numbers $K_{D}^{\star}, K_{P}^{\star}$ and $\theta_{\text {out }}^{\star}$ such that for any $K_{D}<K_{D}^{\star}, K_{P}>K_{P}^{\star}$ and $\left\|\theta_{\text {out }}\right\|_{\infty}<\theta_{\text {out }}^{\star}$ there exists a suitable choice of the design parameters $K_{i}, \lambda_{i}, i=\{1,2\}$, dependent only on $\theta_{\text {out }}^{\star}$, that renders the closed-loop system ISS with restrictions $R_{z}$ and $R_{\theta}$ on the initial states $\|(\tilde{z}(0), \dot{\tilde{z}}(0))\|$ and $\|(\tilde{\theta}(0), \dot{\tilde{\theta}}(0))\|$, no restrictions on the initial state $\|(\tilde{x}(0), \dot{\tilde{x}}(0))\|$ and restrictions $\left(R_{\delta_{z}}, \epsilon R_{\delta_{x}}\right)$ on the inputs $\left(\delta_{x}, \delta_{z}\right)$.

\section{SEnsor NoIse RobUSTnESS}

The control methodology developed in the previous sections relies on a perfect identification of the current operating mode and assumes the availability of discontinuous control inputs. Given the nature of $\mathcal{G}(\{T L, F F\})$ and $\mathcal{G}(\{F F, T L\})$, the vehicle can be $\epsilon$-robustly in the TL or FF operating mode at any time by appropriately setting the controls, independently of the current operating mode. However, this situation can generate large abrupt changes in control authority which are not realistic. We now prove that the hybrid controller is stable and robust to measurement noise on the force sensor readings, without large instantaneous variations of control, by showing that the FF control law can stabilize both the TL and FF operating modes. When in the region of influence of the sensor noise we can use this controller to stabilize the system without knowledge of the current operating mode.

Let $\hat{F}_{Z}$ be the sensor reading for the vertical force and $\sigma_{1}$ be its maximum error, with $\sigma_{2}>\sigma_{1}>0$. For measurements $\hat{F}_{Z}<-\sigma_{2}$ the local controller selected is the TL controller and once $\hat{F}_{Z} \geq-\sigma_{2}$ is attained we select the FF controller. This law for the supervisor controller guarantees that the TL controller is never used when the vehicle is in the FF operating mode. The desired trajectory tracking behaviour is achieved if the conditions from Theorem 3 are verified. Additionally, no chattering is observed provided that once the change from TL to FF controller is performed, the FF controller is applied until the vehicle is robustly in the FF operating mode.

Assume that the FF controller is being used when the vehicle is in the TL mode. In that situation, the common mode input is given by

$$
u=m g-m \ddot{z}^{\star}-k_{1} \tilde{z}-k_{2} \dot{\tilde{z}} .
$$

The idea is to show that the FF control law is still able to achieve practical stability of the attitude dynamics, avoiding 
undesired overturns, so that in finite time the quadrotor will be able to takeoff. For this reason we now focus on the angle dynamic equation. Departing from (9), substituting $F_{\theta}$ as a function of the FF controls $u, v$, and using (18) and (1) we can express the dynamics equation for the time evolution of $\theta$ as

$$
\begin{aligned}
\ddot{\theta}= & \frac{v r+u\left(\ell_{g}-\ell \sin \theta \sin (\theta+\gamma)\right)}{m \ell^{2} \cos ^{2}(\theta+\gamma)}+\tan (\theta+\gamma) \dot{\theta}^{2} \\
& -\frac{g}{\ell \cos (\theta+\gamma)}+\frac{\lambda_{H} \tan (\theta+\gamma)}{m \ell \cos (\theta+\gamma)} \dot{x}-\frac{\lambda_{H} \tan (\theta+\gamma)}{m \ell \cos (\theta+\gamma)} \sin (\theta) \dot{\theta}
\end{aligned}
$$

Consider a transition maneuver where the angle $\theta$ is constant, resulting in $\dot{\theta}^{\star}=0, \ddot{\theta}^{\star}=0$ and $v^{\star}=0$. With the free flight controller (17), considering $\lambda_{H}=0$, and noting that $\ell_{g}=\ell \cos (\theta+\gamma)$, the resulting closed-loop tracking error dynamic equation for the angle is

$$
\begin{aligned}
& \ddot{\tilde{\theta}}=\frac{1}{m \ell^{2} \cos ^{2}(\theta+\gamma)}\left(-r K_{P}\left(K_{D} \dot{\tilde{\theta}}+\tan \left(\tilde{\theta}+\theta^{\star}\right)-\tan \theta^{\star}\right)\right. \\
& \left.+m \ell^{2} \sin (\theta+\gamma) \cos (\theta+\gamma) \dot{\tilde{\theta}}^{2}+v_{\Delta}\left(\theta_{\text {out }}, \tilde{z}, \dot{\tilde{z}}, \dot{z}^{\star}, \ddot{z}^{\star}\right)\right)
\end{aligned}
$$

with

$v_{\Delta}(\cdot)=-r K_{P} K_{D} \theta_{\text {out }}+\ell\left(m \ddot{z}^{\star}+\lambda_{z 0} \dot{z}^{\star}-k_{1} \tilde{z}-k_{2} \dot{\tilde{z}}\right) \cos (\theta+\gamma)$.

Theorem 3: Consider the attitude error dynamics (19). Let be $K_{D}$ fixed as in Proposition 2. Then there exist $\Delta, \Delta_{v}$ and, $K_{P 2}^{\star}$ such that for all $K_{P}>K_{P 2}^{\star}$ system (19) is ISS with respect to the exogenous input $v_{\Delta}$ with restrictions $\Delta_{v}$ on the input and $\Delta$ on the initial states $\|(\tilde{\theta}(0), \dot{\tilde{\theta}}(0))\|$.

According to the above proposition the free flight control law is tuned by fixing the parameters $K_{D}, K_{i}, \lambda_{i}, i=\{1,2\}$ as suggested in Proposition 2, while the proportional gain $K_{P}$ is chosen such that $K_{P}>\max \left\{K_{P}^{\star}, K_{P 2}^{\star}\right\}$, in a way which turns out to be fully compatible with both Proposition 2 and Theorem 3.

\section{Simulation Results}

In this section we present the results from a simulation run of the proposed controller. The reference trajectory consists of a $\mathrm{TL} \rightarrow \mathrm{FF}$ robust transition maneuver. The tilt angle $\theta$ is first driven to a reference value by generating torque around the contact point $P_{2}$ and then the reference horizontal acceleration is augmented to drive the quadrotor to the free flight operating mode. The vehicle parameters are $m=1 \mathrm{~kg}$, $J=0.5 \mathrm{~kg} \mathrm{~m}^{2}, l_{g}=0.3 \mathrm{~m}, \gamma=10^{\circ}, r=0.5 \mathrm{~m}, \beta=1 \mathrm{~m}$, $\lambda_{x}=1, \lambda_{x 0}=0.5, \lambda_{z}=1, \lambda_{z 0}=0.5, \lambda_{H}=10, \lambda_{H 0}=8$. The controller parameters are $K_{P}=40, K_{D}=2$ for the TL mode and $K_{1}=2, K_{2}=1, \lambda_{1}=0.2, \lambda_{2}=3, K_{P}=70$, $K_{D}=1, k_{1}=10, k_{2}=10$ for the FF operating mode.

In both figures the vertical dashed line around the 1.2 seconds mark indicated the time instant when the change of operating mode from TL to FF occurs. Figure 2(a) presents the time evolution of the centre of mass $(x, z)$ coordinates during the takeoff maneuver. The quadrotor horizontal position is initially almost constant but follows a quadratic evolution once a reasonable tilt angle is achieved, approximately around the 0.5 second mark. The height of the UAV follows the tilt angle and has little variation during the TL operating mode. Once the free flight mode is attained the height follows a quadratic time evolution. It can be seen that the vehicle follows closely the desired trajectory.

Figure 2(b) shows the actual and reference actuations during the maneuver. The small actuation jump around the 0.8 seconds mark occurs due to the switch from the $\theta$ to the $(\alpha, \theta)$ controller while in the TL mode. When in free flight the forces are almost identical, again due to the fact that we are tracking reference trajectories of constant horizontal and vertical acceleration, leading to a slowly varying reference angle.

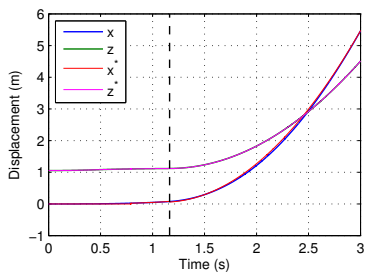

(a)

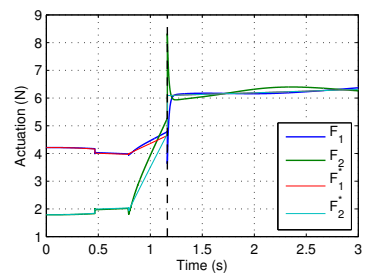

(b)
Fig. 2. (a) Planar quadrotor center of mass coordinates. (b) Planar quadrotor $F_{1}$ and $F_{2}$ actuations

\section{CONCLUSIONS}

This paper addressed the problem of robust takeoff and landing control of a quadrotor UAV. A controller was designed that presents a solution to the takeoff and landing problem and achieves robustness to external disturbances and uncertainties, in both the environment and the vehicle dynamics. Robustness to measurement noise in the detection of the operating mode of the hybrid model was also achieved by the proposed controller. Simulation results were presented to assess the performance of the proposed controller.

\section{REFERENCES}

[1] "Civilian applications: the challenges facing the uav industry," Air \& Space Europe, 1999.

[2] T. Samad, J. Bay, and D. Godbole, "Network-centric systems for military operations in urban terrain: The role of uavs," Proceedings of the IEEE, vol. 95, no. 1, pp. 92-107, Jan. 2007.

[3] K. Ro, J.-S. Oh, and L. Dong, "Lessons learned: Application of small uav for urban highway traffic monitoring," 45th AIAA Aerospace Sciences Meeting and Exhibit, 2007.

[4] J. M. Sullivan, "Revolution or evolution? the rise of the uavs," 45th AIAA Aerospace Sciences Meeting and Exhibit, 2007.

[5] G. Zhou and D. Zang, "Civil uav system for earth observation," Geoscience and Remote Sensing Symposium, 2007.

[6] L. Marconi, R. Naldi, and L. Gentili, "A control framework for robust practical tracking of hybrid automata," Conference on Decision and Control, 2009.

[7] C. Cai, R. Goebel, R. G. Sanfelice, and A. R. Teel, Hybrid Systems: Limit Sets and Zero Dynamics with a View Toward Output Regulation. Springer Berlin Heidelberg, 2008.

[8] M. Heymann, F. Lin, G. Meyer, and S. Resmerita, "Analysis of zeno behaviors in a class of hybrid systems," Automatic Control, IEEE Transactions on, vol. 50, no. 3, pp. 376-383, March 2005.

[9] M. Lazar and W. Heemels, "Predictive control of hybrid systems: Input-to-state stability results for sub-optimal solutions," Automatica, vol. 45 , no. 1 , pp. $180-185,2009$.

[10] R. Goebel and A. Teel, "Solutions to hybrid inclusions via set and graphical convergence with stability theory applications," Automatica, vol. 42 , no. 4 , pp. $573-587,2006$.

[11] R. Goebel, R. Sanfelice, and A. Teel, "Hybrid dynamical systems," Control Systems Magazine, IEEE, vol. 29, no. 2, pp. 28-93, April 2009.

[12] A. Isidori, L. Marconi, and A. Serrani, Robust Autonomous Guidance. Springer, 2003.

[13] A. Isidori, Nonlinear Control Systems II. Springer Verlag London, 1999. 